\title{
El mosquito del dengue en la Ciudad de México. Invasión incipiente de Aedes aegypti y sus potenciales riesgos
}

\author{
María D. Mejía-Guevara, ${ }^{1}$ Fabián Correa-Morales, ${ }^{2}$ Cassandra González-Acosta, ${ }^{3}$ \\ Eduardo Dávalos-Becerril, ${ }^{2}$ Jorge L. Peralta-Rodríguez, ${ }^{4}$ Andrés Martínez-Gaona, ${ }^{4}$ \\ Mariela Hernández-Nava, ${ }^{4}$ Carlos Ramírez-Huicochea, ${ }^{4}$ Leopoldo Rosas-Trinidad, ${ }^{4}$ \\ Mariana Carmona-Pérez, ${ }^{3}$ Víctor Salazar-Bueyes, ${ }^{2}$ Fernando Tapia-Olarte ${ }^{1}$ y Miguel Moreno-García ${ }^{4}$
}

${ }^{1}$ Servicios de Salud Pública de la Ciudad de México, Subdirección de Vigilancia y Evaluación Epidemiología, Ciudad de México; ${ }^{2}$ Centro Nacional de Programas Preventivos y Control de Enfermedades, Ciudad de México; ${ }^{3}$ Servicios de Salud de Morelos, Coordinación de Enfermedades Transmitidas por Vector y Zoonosis, Morelos; ${ }^{4}$ Servicios de Salud de Morelos, Centro Regional de Control de Vectores Panchimalco, Unidad de Investigación Entomológica y Bioensayos, Morelos. México

\section{Resumen}

Introducción: La Ciudad de México no tiene presencia endémica de Aedes aegypti, por lo que está libre de enfermedades transmitidas por vector como dengue, Zika y chikunguña. Sin embargo, existe evidencia de la presencia de huevecillos en la urbe desde 2015. Objetivo: Reportar la presencia constante y en aumento de huevecillos de Aedes aegypti en la Ciudad de México de 2015 a 2018. Método: Se realizó vigilancia a través de ovitrampas; se contabilizaron y eclosionaron huevecillos para determinar la especie. Resultados: De 2015 a 2018 fueron identificados 378 organismos como Aedes aegypti. En total fueron colectadas 76 ovitrampas positivas a Aedes aegypti en 50 sitios distintos de 11 alcaldías. El noreste de la Ciudad de México fue el área con mayor positividad. Conclusiones: Los resultados pueden estar indicando un periodo de colonización incipiente y la probable la existencia de colonias crípticas del mosquito, por lo que la Ciudad de México podría estar en riesgo de presentar epidemias de enfermedades transmitidas por vector.

PALABRAS CLAVE: Aedes aegypti. Ciudad de México. Enfermedades transmitidas por vector. Mosquitos. Vigilancia entomológica.

\section{Aedes aegypti, the dengue fever mosquito in Mexico City. Early invasion and its potential risks}

\section{Abstract}

Introduction: Mexico City has no endemic presence of Aedes aegypti, and it is therefore free of vector-borne diseases, such as dengue fever, Zika and chikungunya. However, evidence has shown the presence of Aedes aegypti eggs in the city since 2015. Objective: To report the constant and increasing presence of Aedes aegypti eggs in Mexico City from 2015 to 2018. Methods: Surveillance was carried out using ovitraps. Eggs were counted and hatched in order to determine the species. Results: From 2015 to 2018, 378 organisms were identified as Ae. aegypti. In total, 76 Aedes aegypti-positive ovitraps were collected at 50 different places in 11 boroughs of the city. Northeastern Mexico City was the area with the highest number of positive traps. Conclusions: The results may be indicating a period of early colonization and the probable existence of cryptic colonies of the mosquito, and Mexico City could be therefore at risk of experiencing vector-borne epidemics.

KEY WORDS: Aedes aegypti. Mexico City. Vector-borne diseases. Mosquitoes Entomological surveillance.

Correspondencia:

*Miguel Moreno-García

E-mail: miguelmoga2000@yahoo.com.mx
Gac Med Mex. 2020;156:388-395

Disponible en PubMed

www.gacetamedicademexico.com

0016-3813/๑ 2020 Academia Nacional de Medicina de México, A.C. Publicado por Permanyer. Este es un artículo open access bajo la licencia CC BY-NC-ND (http://creativecommons.org/licenses/by-nc-nd/4.0/). 


\section{Introducción}

El mosquito Aedes aegypti (orden Diptera, familia Culicidae, subgénero Stegomyia), descrito originalmente como Culex aegypti por Carl Linnaeus en 1792, es el principal vector transmisor de arbovirus (contracción de arthropod-borne virus) como los causantes de la fiebre amarilla, dengue, Zika y chikunguña. Las enfermedades ocasionadas por estos arbovirus se les denomina enfermedades transmitidas por vector (ETV). Actualmente, 30 estados de México reportan casos de ETV endémicas; Tlaxcala y la Ciudad de México (CDMX) son los únicos estados libres de ETV.

La CDMX se encuentra a una altitud de $2240 \mathrm{~m}$ y su rango de temperatura promedio anual es de -2 a $28{ }^{\circ} \mathrm{C}$. Presenta climas templado-subhúmedo, semifrío-subhúmedo-húmedo, semiseco templado; así como lluvias estacionales en el verano, con una precipitación anual promedio de $720 \mathrm{~mm} .^{1,2}$ Por estas características geográficas y climáticas, constituía una zona con poca probabilidad para el establecimiento de Aedes aegypti, especie adaptada a climas tropicales y subtropicales. Sin embargo, evidencias recientes muestran la presencia intermitente del mosquito en la ciudad. ${ }^{3,4}$

Existen factores causales para la introducción y colonización de Aedes aegypti: la continua urbanización (legal e ilegal), la mala condición y hacinamiento de las viviendas, la insuficiencia de acceso al agua potable y al alcantarillado y la deficiente gestión de residuos; el cambio climático y el incremento de temperatura del agua y aire en áreas urbanas;, ${ }^{5,6}$ así como la movilidad de los individuos desde lugares con presencia permanente del mosquito, lo cual puede inducir una dispersión del vector y del virus., ${ }^{7,8} \mathrm{La}$ interacción de estos factores coloca a la CDMX en riesgo de convertirse en un área donde los mosquitos (y los arbovirus que transmiten) puedan establecerse.

Debido a que la CDMX está rodeada por estados con presencia permanente de Aedes aegypti y ETV endémicas (Estado de México y Morelos) y presenta gran movilidad humana y de transporte foráneo, así como aumento en la temperatura atmosférica (Figura 1), desde 2009 se estableció el uso de ovitrampas para el monitoreo del mosquito. Las ovitrampas son recipientes obscuros, cuyo interior se encuentra recubierto por un sustrato de oviposición y se llena con agua;' ${ }^{2}$ simulan los lugares donde el mosquito pone sus huevecillos y constituyen una herramienta para el monitoreo de Aedes aegypti y otros mosquitos. ${ }^{10-12} \mathrm{El}$ primer registro de huevecillos de Aedes aegypti en la CDMX se llevó a cabo en 2015 ; $^{3}$ a partir de entonces, se ha detectado un incremento constante en el número de ovitrampas con huevecillos del mosquito. En este artículo se reporta la presencia del mosquito detectada en ovitrampas de 2015 a 2018, se discute el potencial establecimiento de Aedes aegypti en la CDMX y el posible riesgo que representa para la salud pública.

\section{Método}

Las colectas se realizaron conforme la Guía Metodológica del Centro Nacional de Programas Preventivos y Control de Enfermedades. ${ }^{9}$ Las ovitrampas fueron recipientes plásticos negros de $1 \mathrm{~L}$ de capacidad. Como sustrato de ovoposición se usaron hojas de tela pellón (F-1600) de $12 \mathrm{~cm}$ de ancho por $35 \mathrm{~cm}$ de largo, con las que se recubrieron los interiores de las ovitrampas, las cuales posteriormente fueron llenadas con agua, sin llegar al borde superior para dejar expuestos 2 a $3 \mathrm{~cm}$ de las hojas (área donde las hembras colocan sus huevecillos), y colocadas en lugares sombreados, a menos de $1.5 \mathrm{~m}$ de altura. Las ovitrampas eran revisadas cada siete días y se retiraban las hojas con huevecillos, en las cuales se depositaron los huevecillos que se encontraban en el agua 0 en el fondo de las ovitrampas, las cuales fueron lavadas, recubiertas con nuevas hojas de tela, rellenadas con agua limpia y colocadas nuevamente en el mismo sitio.

Las hojas de peyón se enviaron a la Unidad de Investigación Entomológica y Bioensayos del Centro Regional de Control de Vectores Panchimalco, en Morelos, donde se realizó el conteo, eclosión de los huevecillos y determinación taxonómica de los organismos. Para la eclosión, las hojas fueron sumergidas en agua en charolas plásticas con volumen de $1 \mathrm{~L}$. Las larvas eclosionadas se alimentaron con croquetas para rata. Los adultos emergidos se colocaron en jaulas de malla plástica y fueron alimentados ad libitum con solución azucarada. La temperatura de crianza fue de $29 \pm 2{ }^{\circ} \mathrm{C}$, con 60 a $70 \%$ de humedad relativa y periodos de luz-oscuridad natural. Las larvas fueron determinadas taxonómicamente durante el tercer y cuarto estadio de desarrollo. Para la estratificación de las larvas y los especímenes adultos se usó la clave taxonómica Ibáñez-Bernal y MartínezCampo $^{13}$ y Darsie y Ward. ${ }^{14}$ 

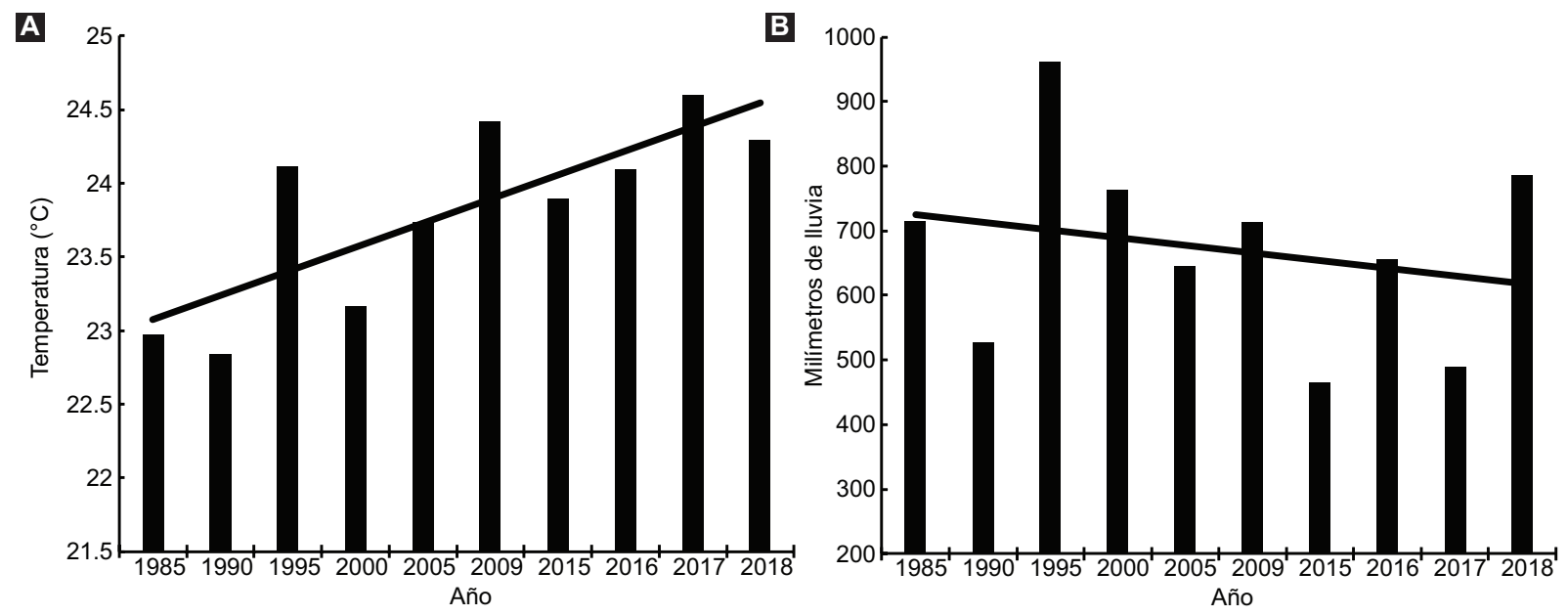

Figura 1. A) Temperatura máxima promedio anual y precipitación $(\mathrm{mm})$ anual histórica de la Ciudad de México. Datos obtenidos de https://smn. cna.gob.mx/es/climatologia/temperaturas-y-lluvias/resumenes-mensuales-de-temperaturas-y-lluvias.

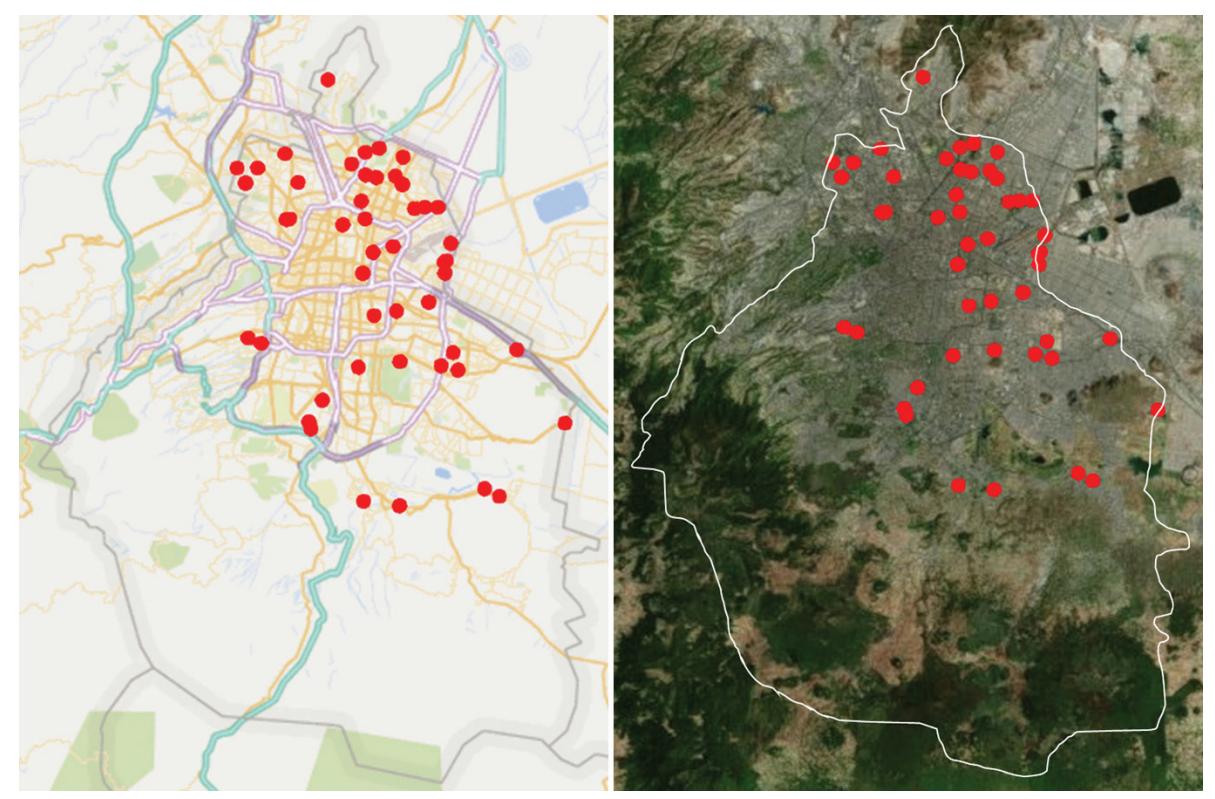

Figura 2. Ubicación de los sitios de colecta de ovitrampas positivas a Aedes aegypti en la Ciudad de México (vista en mapa urbano y satelital).

Las ovitrampas se colocaron en zonas públicas no residenciales como jardines, plazas y parques públicos, reservas naturales dentro de área urbana, avenidas y camellones. También se incluyeron áreas externas con libre acceso de centros de salud, escuelas, iglesias, mercados, unidades deportivas o de recreación, centros de convivencia social, terminales de autobuses foráneos y embarcaderos (Xochimilco). Anualmente se colocaron 2240 ovitrampas, excepto en 2015 cuando se emplearon 1440, y se abarcaron las 16 alcaldías de la CDMX.

\section{Resultados}

De las colectas realizadas entre 2015 y 2018 se detectaron 1531 ovitrampas con huevecillos, colectadas en 358 distintos sitios. El total de huevecillos fue de 48 477, de los cuales 16171 eclosionaron; 378 fueron Aedes aegypti, que representaron $0.77 \%$ de éxito de eclosión. En total, 76 ovitrampas positivas a Aedes aegypti fueron colectadas en 50 sitios distintos de 11 alcaldías (Figura 2 y Tabla 1). El mayor número de larvas fue colectado en septiembre (época de 
Tabla 1. Sitios de la Ciudad de México en los que se colectó Aedes aegypti mediante ovitrampas entre 2015 y 2018

\begin{tabular}{|c|c|c|c|c|c|c|}
\hline Año & Alcaldía & Sitio & Latitud & Longitud & Mes & Núm. larvas \\
\hline \multirow[t]{2}{*}{2015} & Azcapotzalco & Tren suburbano (Pantaco) & 19.479908 & -99.168761 & Septiembre & 14 \\
\hline & Gustavo A. Madero & Casa del Peregrino & 19.483314 & -99.109786 & Septiembre & 4 \\
\hline \multirow[t]{3}{*}{2016} & Venustiano Carranza & Alameda Oriente & 19.436358 & -99.053873 & Septiembre & 1 \\
\hline & Venustiano Carranza & Conalep Aeropuerto & 19.424043 & -99.057283 & - & 1 \\
\hline & Venustiano Carranza & TAPO & 19.42989 & -99.112319 & - & 1 \\
\hline \multirow[t]{32}{*}{2017} & Álvaro Obregón & Liga de Béisbol Maya & 19.364826 & -99.196399 & Septiembre & 14 \\
\hline & Álvaro Obregón & Parque Valentín G. Farías & 19.368711 & -99.206424 & Septiembre & 1 \\
\hline & Azcapotzalco & Alameda Norte & 19.500219 & -99.178502 & Septiembre & 1 \\
\hline & Azcapotzalco & Deportivo Renovación Nacional & 19.490113 & -99.214643 & Septiembre & 2 \\
\hline & Azcapotzalco & Jardín Margarita Maza de Juárez & 19.490282 & -99.199102 & Noviembre & 1 \\
\hline & Azcapotzalco & Parque Chilapillas & 19.479363 & -99.208156 & Agosto & 1 \\
\hline & Coyoacán & Deportivo Santa Úrsula & 19.308975 & -99.160651 & Octubre & 8 \\
\hline & Coyoacán & Deportivo Santa Úrsula & 19.308975 & -99.160651 & Septiembre & 2 \\
\hline & Coyoacán & Parque las Montañas & 19.347868 & -99.123349 & Noviembre & 2 \\
\hline & Coyoacán & Parque las Montañas & 19.347868 & -99.123349 & Octubre & 6 \\
\hline & Coyoacán & Parque Santa Úrsula & 19.303734 & -99.159131 & Junio & 1 \\
\hline & Cuauhtémoc & Jardín Santiago & 19.449623 & -99.135017 & Septiembre & 1 \\
\hline & Gustavo A. Madero & Basílica de Guadalupe & 19.485171 & -99.117862 & Octubre & 1 \\
\hline & Gustavo A. Madero & Bosque de San Juan de Aragón & 19.462061 & -99.073349 & Septiembre & 1 \\
\hline & Gustavo A. Madero & Deportivo Carmen Serdán & 19.553124 & -99.146329 & Julio & 1 \\
\hline & Gustavo A. Madero & Deportivo Heberto Castillo & 19.497863 & -99.089899 & Junio & 8 \\
\hline & Gustavo A. Madero & Deportivo Luiggy & 19.484424 & -99.095655 & Agosto & 1 \\
\hline & Gustavo A. Madero & Deportivo Luiggy & 19.484424 & -99.095655 & Julio & 4 \\
\hline & Gustavo A. Madero & Deportivo Luiggy & 19.484424 & -99.095655 & Septiembre & 2 \\
\hline & Gustavo A. Madero & Deportivo Miguel Alemán & 19.493173 & -99.128598 & Septiembre & 8 \\
\hline & Gustavo A. Madero & Parque Corpus Christi Norte & 19.46648 & -99.121144 & Agosto & 2 \\
\hline & Gustavo A. Madero & Parque Corpus Christi Norte & 19.46648 & -99.121144 & Julio & 1 \\
\hline & Gustavo A. Madero & Parque Recreativo Justicia Social & 19.478533 & -99.090386 & Agosto & 4 \\
\hline & Gustavo A. Madero & Parque Recreativo Justicia Social & 19.478533 & -99.090386 & Agosto & 1 \\
\hline & Gustavo A. Madero & Parque Recreativo Justicia Social & 19.478533 & -99.090386 & Noviembre & 5 \\
\hline & Gustavo A. Madero & Parque Recreativo Justicia Social & 19.478533 & -99.090386 & Septiembre & 2 \\
\hline & Gustavo A. Madero & Parque Recreativo Justicia Social & 19.478533 & -99.090386 & Septiembre & 1 \\
\hline & Gustavo A. Madero & Rancho Grande la Villa & 19.501382 & -99.118285 & Julio & 7 \\
\hline & Gustavo A. Madero & Reserva de Tepeyac & 19.504375 & -99.107777 & Julio & 3 \\
\hline & Gustavo A. Madero & Reserva de Tepeyac & 19.504375 & -99.107777 & Junio & 2 \\
\hline & Gustavo A. Madero & Reserva de Tepeyac & 19.504375 & -99.107777 & Noviembre & 21 \\
\hline & Gustavo A. Madero & Reserva de Tepeyac & 19.504375 & -99.107777 & Noviembre & 5 \\
\hline
\end{tabular}


Tabla 1. Sitios de la Ciudad de México en los que se colectó Aedes aegypti mediante ovitrampas entre 2015 y 2018 (Continuación)

\begin{tabular}{|c|c|c|c|c|c|c|}
\hline Año & Alcaldía & Sitio & Latitud & Longitud & Mes & Núm. larvas \\
\hline & Gustavo A. Madero & Reserva de Tepeyac & 19.504375 & -99.107777 & Octubre & 7 \\
\hline & Gustavo A. Madero & Zoológico de San Juan de Aragón & 19.461268 & -99.081155 & Noviembre & 1 \\
\hline & Gustavo A. Madero & Zoológico de San Juan de Aragón & 19.461268 & -99.081155 & Septiembre & 10 \\
\hline & Iztacalco & $\begin{array}{l}\text { Centro de Salud TIII Dr. Manuel } \\
\text { Pesqueira }\end{array}$ & 19.394339 & -99.070505 & Agosto & 1 \\
\hline & Iztacalco & Deportivo Metro & 19.387966 & -99.094784 & Septiembre & 2 \\
\hline & Iztacalco & Parque el Foro & 19.3848 & -99.111596 & Julio & 1 \\
\hline & Iztapalapa & Alberca Salvador Allende & 19.358232 & -99.052353 & Agosto & 1 \\
\hline & Iztapalapa & Biblioteca Antonio Gómez Rodríguez & 19.348838 & -99.061293 & Julio & 1 \\
\hline & Iztapalapa & Centro Social Villa Estrella & 19.351701 & -99.092109 & Octubre & 102 \\
\hline & Iztapalapa & Centro Deportivo Atlético Mexicano & 19.374861 & -99.063031 & Septiembre & 1 \\
\hline & Iztapalapa & Deportivo Santa Cruz Meyehualco & 19.345768 & -99.048541 & Julio & 2 \\
\hline & Iztapalapa & Deportivo Santa Cruz Meyehualco & 19.345768 & -99.048541 & Septiembre & 1 \\
\hline & Iztapalapa & Jardín Francisco J. Mujica & 19.349013 & -99.061294 & Septiembre & 10 \\
\hline & Miguel Hidalgo & CECYT 9 & 19.453899 & -99.175013 & Octubre & 1 \\
\hline & Miguel Hidalgo & CECYT 9 & 19.453899 & -99.175013 & Septiembre & 1 \\
\hline & Miguel Hidalgo & Jardín Cañitas & 19.45346 & -99.177568 & Julio & 4 \\
\hline & Tláhuac & Centro de salud de Santa Catarina & 19.307826 & -98.968109 & Julio & 2 \\
\hline & Venustiano Carranza & Conalep Venustiano Carranza II & 19.422892 & -99.05879 & Septiembre & 1 \\
\hline & Venustiano Carranza & Deportivo Lázaro Cárdenas & 19.414801 & -99.119973 & Julio & 1 \\
\hline & Venustiano Carranza & Deportivo Lázaro Cárdenas & 19.415124 & -99.11997 & Noviembre & 22 \\
\hline & Venustiano Carranza & Deportivo Lázaro Cárdenas & 19.414801 & -99.119973 & Octubre & 4 \\
\hline & Venustiano Carranza & Deportivo Moctezuma & 19.434247 & -99.097297 & Octubre & 3 \\
\hline & Venustiano Carranza & Deportivo Plutarco Elías Calles & 19.453841 & -99.118303 & Julio & 26 \\
\hline & Venustiano Carranza & TAPO & 19.42989 & -99.112319 & Junio & 15 \\
\hline & Xochimilco & Casa de Cultura de Tulyehualco & 19.255422 & -99.017332 & Agosto & 1 \\
\hline & Xochimilco & Facultad de Artes y Diseño & 19.251917 & -99.11946 & Septiembre & 1 \\
\hline \multirow[t]{10}{*}{2018} & Coyoacán & Parque Huayamilpas & 19.324223 & -99.150507 & Septiembre & 2 \\
\hline & Gustavo A. Madero & Bosque de San Juan de Aragón & 19.462061 & -99.073349 & Julio & 1 \\
\hline & Gustavo A. Madero & Lienzo Charro de Aragón & 19.462163 & -99.063339 & Julio & 1 \\
\hline & Gustavo A. Madero & Reserva de Tepeyac & 19.504375 & -99.107777 & Julio & 1 \\
\hline & Gustavo A. Madero & Zoológico de San Juan de Aragón & 19.461268 & -99.081155 & Mayo & 1 \\
\hline & Iztacalco & Parque Pantitlán & 19.4151 & -99.058379 & Julio & 1 \\
\hline & Iztapalapa & Alberca Salvador Allende & 19.358232 & -99.052353 & Agosto & 1 \\
\hline & Iztapalapa & Alberca Salvador Allende & 19.358232 & -99.052353 & Julio & 1 \\
\hline & Iztapalapa & Centro Social Villa Estrella & 19.351701 & -99.092109 & Septiembre & 1 \\
\hline & Iztapalapa & Deportivo Santa Martha & 19.360389 & -99.00439 & Septiembre & 1 \\
\hline
\end{tabular}


Tabla 1. Sitios de la Ciudad de México en los que se colectó Aedes aegypti mediante ovitrampas entre 2015 y 2018 (Continuación)

\begin{tabular}{|c|c|c|c|c|c|c|}
\hline Año & Alcaldía & Sitio & Latitud & Longitud & Mes & Núm. larvas \\
\hline & Xochimilco & Casa de Cultura de Tulyehualco & 19.255422 & -99.017332 & Septiembre & 1 \\
\hline & Xochimilco & $\begin{array}{l}\text { Centro de Educación Ambiental } \\
\text { Acuexcomatl }\end{array}$ & 19.260958 & -99.028621 & Julio & 1 \\
\hline & Xochimilco & Embarcadero Zacapa & 19.248801 & -99.092481 & Septiembre & 1 \\
\hline & Xochimilco & Embarcadero Zacapa & 19.248801 & -99.092481 & Septiembre & 1 \\
\hline
\end{tabular}

lluvias), sin embargo, de mayo a noviembre se detectó positividad (Tabla 1).

En más de una ocasión, 16 sitios resultaron positivos y en su mayoría los registros ocurrieron en el norte, noreste y centro de la CDMX (Tabla 1 y Figura 3A). Varios lugares eran zonas con afluencia importante de personas, tanto locales como foráneas, así como zonas turísticas (por ejemplo, Bosque y Zoológico de San Juan de Aragón, Reserva del Tepeyac, estación de autobuses TAPO y Embarcadero Zacapa de Xochimilco). En algunos casos, la positividad se repitió entre años y en otros, la positividad se detectó en distintas colectas durante el mismo año (Tabla 1).

Las ovitrampas positivas se empezaron a identificar en el noreste (2015, 2016 y primer semestre de 2017), área que presenta temperatura de 5 a $28^{\circ} \mathrm{C}$, la mayor de la ciudad (Figura 3B), y un clima templado subhúmedo (de menor humedad)/semiseco templado. No obstante, para el segundo semestre de 2017, el rango de distribución se amplió y abarcó áreas con temperatura de 4 a $24{ }^{\circ} \mathrm{C}$ (clima templado subhúmedo de humedad media). Para 2018 nuevamente se registró la presencia del mosquito en esas zonas (Figuras 3B y 4).

\section{Discusión}

Debido a que en 2015 solo se registraron dos ovitrampas positivas a Aedes aegypti en sitios con gran movilidad humana, se consideró que se trataba de una introducción accidental. Sin embargo, el número de ovitrampas positivas se incrementó en los siguientes tres años, lo que hace suponer que no se debió únicamente al transporte accidental. La falta de recolección de larvas o especímenes adultos, aunada a la baja proporción de huevecillos encontrados y eclosionados, indica que aún no existen poblaciones establecidas de Aedes aegypti, si bien la CDMX está en periodo de colonización incipiente.
La presencia recurrente de ovitrampas positivas en el mismo sitio de colecta podría indicar la posibilidad de que existan colonias crípticas intermitentes del mosquito. Aedes aegypti tiene hábitos domésticos, ${ }^{15,16}$ las hembras tienden a ovipositar en tanques, tambos y pilas, mantenidos con agua para uso doméstico (comúnmente debido a una inadecuada red de agua potable). ${ }^{17}$ Durante la época de lluvias, llantas, botes, cubetas, macetas y cualquier recipiente que pueda contener agua limpia se convierten en lugares idóneos para la oviposición, dentro o fuera del domicilio. ${ }^{17} \mathrm{Sin}$ embargo, ante la escasez de sitios para ovipositar en época de secas, las hembras pueden usar sitios alternos para depositar sus huevecillos como el agua de las cajas de registro, de las alcantarillas de drenaje y tuberías de PVC de estacionamientos subterráneos. ${ }^{18-22}$ Estos sitios tienden a mantener una temperatura cálida estable en áreas sombreadas y con baja evaporación, ${ }^{22}$ por lo que son aptos para albergar colonias de mosquitos. Además, son espacios en los que se dificulta observar las larvas o pupas, convirtiéndose en sitios de riesgo para la CDMX.

Un componente importante para explicar el aumento en el rango de distribución del mosquito es el incremento mundial de la temperatura. ${ }^{23,24}$ En Colombia y Bolivia, el mosquito se ha encontrado a 2302 y $2550 \mathrm{~m}$ sobre el nivel de mar, respectivamente. ${ }^{25,26}$ En México, el mosquito había sido previamente encontrado a una altitud de $2133 \mathrm{~m} .^{27} \mathrm{La}$ presencia en estas altitudes puede estar asociada al aumento de la temperatura. Lo mismo podría estar ocurriendo en la CDMX, cuya temperatura se incrementó en las últimas décadas. El cambio en la temperatura hace que el clima ya no sea una barrera para la dispersión del mosquito.

Los resultados indican mayor positividad en las zonas más calurosas de la ciudad (al noreste), que congregan la mayor carga de población, carecen de abastecimiento regular y adecuado de agua potable y en las que se registra un alto riesgo de inundaciones 


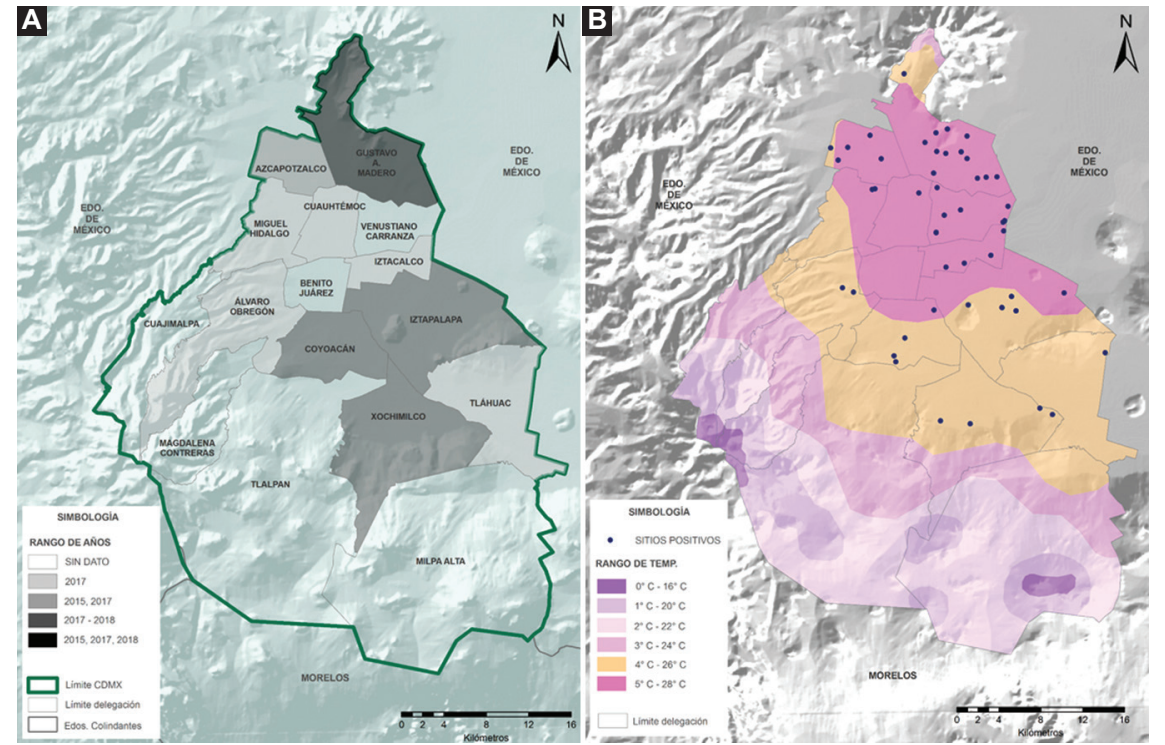

Figura 3. A) Alcaldías con ovitrampas positivas a Aedes aegypti. La alcaldía donde recurrentemente se observaron ovitrampas positivas fue Gustavo A. Madero (2015, 2017, 2018), seguida por Azcapotzalco, Coyoacán, Iztacalco, Venustiano Carranza y Xochimilco, con dos años de colectas positivas. La alcaldía con mayor positividad fue la Gustavo A. Madero seguida por Xochimilco, Venustiano Carranza e Iztapalapa. B) Rangos de temperatura de la CDMX con la ubicación de ovitrampas positivas durante el periodo 2015-2018.
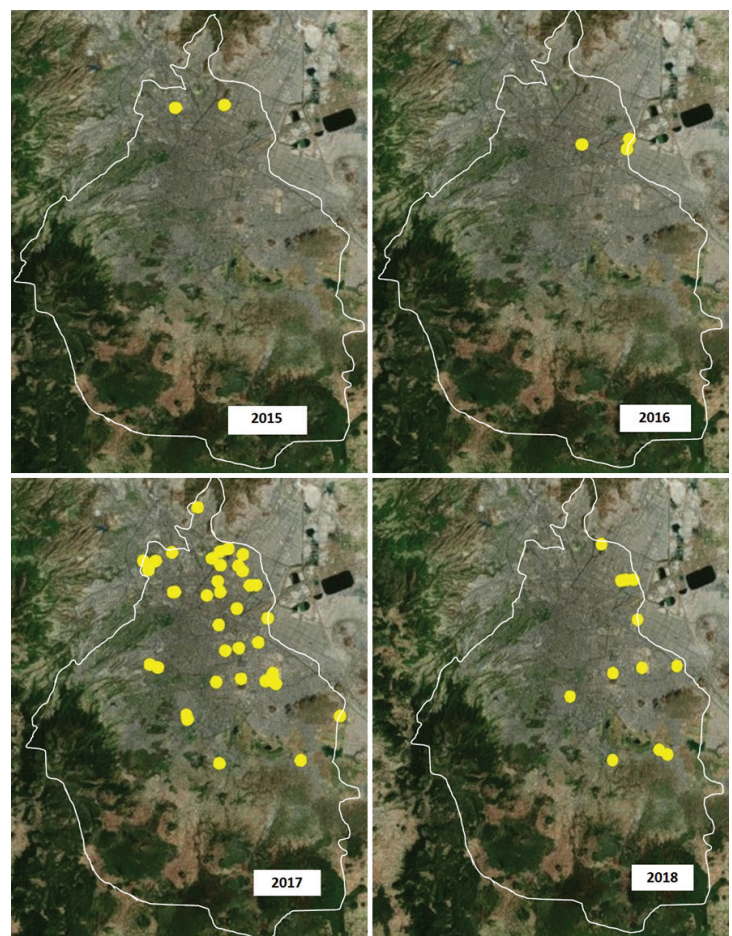

Figura 4. Cronología de la presencia de ovitrampas positivas para Aedes aegypti en zonas urbanas de la Ciudad de México. En el año 2017 se presentó el mayor número de ovitrampas positivas.

durante las temporadas de lluvias. ${ }^{28}$ Además, los asentamientos humanos irregulares (con carencia de servicios públicos) también son comunes en estas áreas. ${ }^{29}$
Aunado a lo anterior, las personas carecen del hábito de la descacharrización (recolección y eliminación de recipientes prescindibles) en patios, alrededores de casas o zonas habitacionales, con lo que contribuyen a la generación de criaderos temporales.

La CDMX está en riesgo de presentar ETV, el cual puede minimizarse mediante la implementación de una adecuada vigilancia, la participación social para la concientización y difusión del conocimiento de las ETV, el mosquito y los métodos de control; así como con la capacitación del personal de salud para que conozca y maneje las guías de práctica clínica para la atención de pacientes. ${ }^{30}$ Con la aplicación de estas estrategias, la CDMX podrá vigilar, limitar y controlar las poblaciones de Aedes aegypti y las ETV asociadas.

\section{Consideraciones éticas}

Para la realización de este estudio, debido a que no se hizo experimentación con humanos o animales, no se necesitaron permisos específicos por parte de las instituciones de adscripción de los autores. Las ubicaciones de colecta no se encuentran en propiedad privada. Los estudios de campo no involucraron especies en peligro de extinción ni protegidas. La Unidad de Investigación Entomológica y BioensayosCentro Regional de Control de Vectores Panchimalco está certificada por el Centro Nacional de Programas 
Preventivos y Control de Enfermedades para la crianza y manutención de colonias de mosquitos.

\section{Agradecimientos}

Agradecemos al personal del área de vectores de Secretaría de Salud de la Ciudad de México, por su asistencia en el trabajo de campo.

\section{Conflicto de intereses}

Los autores declaran no tener conflicto de intereses alguno.

\section{Financiamiento}

Los autores no recibieron patrocinio para llevar a cabo este artículo.

\section{Responsabilidades éticas}

Protección de personas y animales. Los autores declaran que para esta investigación no se han realizado experimentos en seres humanos ni en animales.

Confidencialidad de los datos. Los autores declaran que en este artículo no aparecen datos de pacientes.

Derecho a la privacidad y consentimiento informado. Los autores declaran que en este artículo no aparecen datos de pacientes.

\section{Bibliografía}

1. Instituto Nacional de Estadística y Geografía [Internet]. México: Anuario estadístico y geográfico del Distrito Federal; 2017.

2. Secretaría del Medio Ambiente y Recursos Naturales [Internet]. México: Precipitación media histórica por entidad federativa; 2019.

3. Kuri-Morales P, Correa-Morales F, González-Acosta C, Sánchez-Tejeda G, Dávalos-Becerril E, Juárez-Franco MF, et al. First report of Stegomyia aegypti (= Aedes aegypti) in Mexico City, Mexico. Med Vet Entomol. 2017;31:240-242.

4. Dávalos-Becerril E, Correa-Morales F, González-Acosta C, Santos-Luna R, Peralta-Rodríguez J, Pérez-Rentería C, et al. Urban and semi-urban mosquitoes of Mexico City: a risk for endemic mosquito-borne disease transmission. PLoS One. 2019;14:e0212987.

5. Jansen CC, Beebe NW. The dengue vector Aedes aegypti: what comes next. Microbes Infect. 2010;12:272-279.

6. Li Y, Kamara F, Zhou G, Puthiyakunnon S, Li C, Liu Y, et al. Urbanization increases Aedes albopictus larval habitats and accelerates mosquito development and survivorship. PLoS Negl Trop Dis. 2014;8:e3301.

7. Narro-Robles J, Gómez-Dantés H. El dengue en Mexico: un problema prioritario de salud publica. Salud Publica Mex. 1995;37:12-20.

8. Vázquez-Castellanos J, Canales-Muñoz J, Nápoles-Camacho, Castillo-Morán MA, Ureña-Carillo LE. Estudio del primer gran brote epidémico de dengue en Guadalajara, Jalisco, México, octubre de 1988. Rev Salud Jalisco. 2018;5:8-19.
9. Centro Nacional de Programas Preventivos y Control de Enfermedades [Internet]. Mexico: Guía metodológica para la vigilancia entomológica con ovitrampas; 2017.

10. Fay RW, Eliason DA. A preferred oviposition site as a surveillance method for Aedes aegypti. Mosq News. 1966;26:531-535.

11. Chaverri LG, Dillenbeck C, Lewis D, Rivera C, Romero LM, Chaves LF. Mosquito species (Diptera: Culicidae) diversity from ovitraps in a Mesoamerican tropical rainforest. J Med Entomol. 2018;55:646-653.

12. Ortega-Morales Al, Moreno-García M, González-Acosta C, Correa-Morales $F$. Mosquito surveillance in Mexico: the use of ovitraps for Aedes aegypti, Ae. albopictus, and non-target species. Florida Entomol. 2018;101:623-627.

13. Ibánez-Bernal $S$, Martínez-Campos $C$. Clave para la identificación de larvas de mosquitos comunes en las áreas urbanas y suburbanas de la República Mexicana (Diptera: Culicidae). Folia Entomol Mex. 1994;92:43-73.

14. Darsie RF, Ward RA. Identification and geographical distribution of the mosquitoes of North America, North of Mexico. EE. UU.: University Press of Florida; 2005.

15. Cheong WH. Preferred Aedes aegypti larval habitats in urban areas. Bull World Health Organ. 1967;36:586-589.

16. Candelario-Mejía, G Rodríguez-Rivas A, Muñoz-Urias A, González-Carcamo J, Candelario-Valencia, A Mosso-González C, et al. Estudio observacional de la fluctuación espacial y temporal de Aedes aegypti en el área metropolitana de Guadalajara, México. Rev Med MD. 2014;6:6-12.

17. Villegas-Trejo A, Che-Mendoza A, González-Fernández M, Guillermo-May G, González-Bejarano H, Dzul-Manzanilla F, et al. Control enfocado de Aedes aegypti en localidades de alto riesgo de transmisión de dengue en Morelos, México. Salud Publica Mex. 2011;53:141-151.

18. Marquetti MC, Suárez S, Bisset J, Leyva M. Reporte de hábitats utilizados por Aedes aegypti en Ciudad de La Habana, Cuba. Rev Cubana Med Trop. 2005;57:159-161.

19. De la Cruz-Francisco V, Veda-Moreno DI, Valdés-Murillo A. Aspectos ecológicos de la incidencia larval de mosquitos (Diptera: Culicidae) en Tuxpan, Veracruz, México. Rev Colomb Entomol. 2012;38:128-133.

20. Manrique-Saide P, Arisqueta-Chable C, Geded-Moreno E, Herrera-Bojórquez J, Valentín UC, Chable-Santos $\mathrm{J}$, et al. An assessment of the importance of subsurface catch basins for Aedes aegypti adult production during the dry season in a neighborhood of Merida, Mexico. J Am Mosq Control Assoc. 2013;29:164-167.

21. Arana-Guardia R, Baak-Baak CM, Lorono-Pino MA, Machain-Williams C, Beaty BJ, Eisen L, et al. Stormwater drains and catch basins as sources for production of Aedes aegypti and Culex quinquefasciatus. Acta Trop. 2014; 134:33-42.

22. Unlu I, Faraji A, Indelicato N, Fonseca DM. The hidden world of Asian tiger mosquitoes: immature Aedes albopictus (Skuse) dominate in rainwater corrugated extension spouts. Trans R Soc Trop Med Hyg. 2014;108:699-705.

23. Hopp MJ, Foley JA. Global-scale relationships between climate and the dengue fever vector, Aedes Aegypti. Clim Change. 2001;48:441-463.

24. Kraemer MUG, Sinka ME, Duda KA, Mylne AQN, Shearer FM, Barker CM, et al. The global distribution of the arbovirus vectors Aedes aegypti and Ae. albopictus. Elife. 2015;4:e08347.

25. Ruiz-López F, González-Mazo A, Vélez-Mira A, Gómez GF, Zuleta L, Vélez-Bernal ID. Presencia de Aedes (Stegomyia) aegypti (Linnaeus, 1762) y su infección natural con el virus del dengue en alturas no registradas para Colombia. Biomedica. 2016;36:303-308.

26. Castillo-Quino R, Vallejo-Castro E, Camacho-Aliaga A V, Quiñones-López A, Canelas-Urey HI. Adaptación del mosquito Aedes aegypti a 2550 m s.n.m. Cochabamba, Bolivia. Gac Med Bol. 2018;41:24-30.

27. Lozano-Fuentes S, Hayden MH, Welsh-Rodriguez C, Ochoa-Martínez C Tapia-Santos B, Kobylinski KC, et al. The dengue virus mosquito vector Aedes aegypti at high elevation in México. Am J Trop Med Hyg. 2012;87:902-909.

28. Arellano-Carbajal G, Chaparro-Arias F. Alternativa de suministro de agua potable a la delegación Gustavo A. Madero. [Tesis profesional]. México: Instituto Politecnico Nacional; 2004.

29. Procuraduría Ambiental y del Ordenamiento Territorial del Distrito Federal [Internet]. México: Distribución espacial de los asentamientos humanos irregulares ubicados en el suelo de conservación en relación con el proyecto del Programa General de Ordenamiento Ecológico y Zonas de Valor Ambiental del Distrito Federal; 2011.

30. Fajardo-Dolci G, Meljem-Moctezuma J, Vicente-González E, Venegas-Páez F, Mazón-González B, Aguirre-Gas H. El dengue en México. Conocer para mejorar la calidad de la atención. Rev Med Inst Mex Seguro Soc. 2012;50:631-639. 